\title{
Correction to: Epoetin Biosimilars in the Treatment of Chemotherapy-Induced Anemia: 10 Years' Experience Gained
}

\author{
Matti Aapro $^{1} \cdot$ Andriy Krendyukov $^{2} \cdot$ Martin Schiestl $^{3} \cdot$ Pere Gascón $^{4}$
}

Published online: 28 February 2018

(C) Springer International Publishing AG, part of Springer Nature 2018

\section{Correction to: BioDrugs}

https://doi.org/10.1007/s40259-018-0262-9

Figure 1, HX575 column, 5th box down, which previously read "SC HX575 vs. Eprex ${ }^{\circledR} /$ Erypo $^{\circledR} 417$ patients with CKD-related anemia" as shown here,

The original article can be found online at https://doi.org/10.1007/ s40259-018-0262-9.

Andriy Krendyukov

andriy.krendyukov@sandoz.com

1 Institut Multidisciplinaire d'Oncologie, Clinique de Genolier, Genolier, Switzerland

2 Hematology/Nephrology, Sandoz Biopharmaceuticals, HEXAL AG, Industriestr. 25, 83607 Holzkirchen, Germany

3 Sandoz Biopharmaceuticals, Sandoz International GmbH, Kundl, Austria

4 Department of Hematology-Oncology, Hospital Clínic de Barcelona, University of Barcelona, Barcelona, Spain 
SB309

\begin{tabular}{|c|c|}
\hline $\begin{array}{c}\text { Phase I PK/PD } \\
\text { studies }\end{array}$ & $\begin{array}{c}\text { Eprex } \\
24 \text { healthy subjects }\end{array}$ \\
\hline SC SB309 vs. \\
Eprex ${ }^{\circledR} /$ Erypo $^{\circledR}$ \\
48 healthy subjects
\end{tabular}

Confirmatory phase III studies

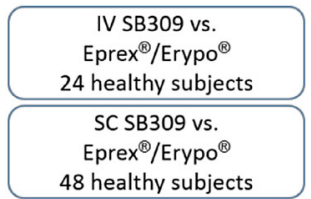

IV SB309 vs.

Eprex ${ }^{\circledR} /$ Erypo $^{\circledR}$

402 HD patients with

CKD-related anaemia

IV SB309 vs.

Eprex ${ }^{\circledast} /$ Erypo $^{\circledR}$

609 HD patients with

CKD-related anemia

Long-term extension

study of patients with

renal anemia in Phase III studies $(n=745)$

Uncontrolled safety study

in patients cancer and $\mathrm{CIA}(n=216)$

\section{HX575}

\begin{tabular}{|c|}
\hline $\begin{array}{c}\text { IV HX575 vs. } \\
\text { Eprex }^{\circledR} / \text { Erypo }^{\circledR} \\
80 \text { healthy subjects }\end{array}$ \\
\hline $\begin{array}{c}\text { IV HX575 vs. } \\
\text { Eprex }^{\circledR} / \text { Erypo }^{\circledR} \\
80 \text { healthy subjects }\end{array}$ \\
\hline $\begin{array}{c}\text { SC HX575 vs. } \\
\text { NeoRecormon }{ }^{\circledast} \\
80 \text { healthy subjects }\end{array}$ \\
\hline
\end{tabular}

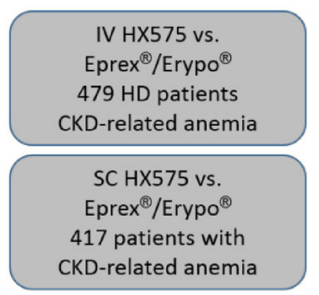

SC HX575 vs.

Eprex ${ }^{\left({ }^{2}\right.} /$ Erypo $^{(8)}$

114 patients with cancer and $\mathrm{CIA}$

should read "SC HX575 417 patients with CKD-related anemia" as shown below.

SB309

Phase I PK/PD studies

Confirmatory

phase III studies

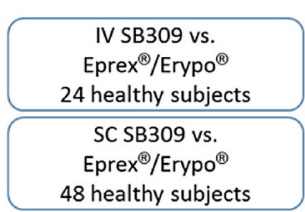

IV SB309 vs. Eprex $^{\circledR 8} /$ Erypo $^{\circledR}$ 402 HD patients with CKD-related anaemia

IV SB309 vs. Eprex $^{\circledR} /$ Erypo $^{\circledR}$ $609 \mathrm{HD}$ patients with CKD-related anemia

Long-term extension study of patients with renal anemia in Phase III studies $(n=745)$

Uncontrolled safety study in patients cancer and $\mathrm{CIA}(n=216)$

\section{HX575}

IV HX575 vs. Eprex $^{\circledR} /$ Erypo $^{\circledR}$ 80 healthy subjects

IV HX575 vs. Eprex ${ }^{\circledR} /$ Erypo $^{\circledR}$ 80 healthy subjects

SC HX575 vs.

NeoRecormon ${ }^{\circledR}$

80 healthy subjects

IV HX575 vs.

Eprex ${ }^{\circledR} /$ Erypo $^{\circledR}$

479 HD patients

CKD-related anemia

SC HX575

417 patients with CKD-related anemia

SC HX575 vs.

Eprex ${ }^{\circledR} /$ Erypo $^{(3)}$

114 patients with cancer and $\mathrm{CIA}$ 